\title{
Hábitat III: \\ La participación de la sociedad civil en la construcción de la Nueva Agenda Urbana y el derecho a la ciudad* \\ Javier Dávalos González**
}

\section{Introducción}

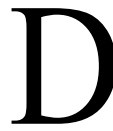
urante la Conferencia Hábitat III, celebrada en la ciudad de Quito en octubre de 2016, fue aprobada la Nueva Agenda Urbana (NAU). Este documento establece la necesidad de construir ciudades sostenibles, resilientes e inclusivas. La NAU establece el ideal de una ciudad para todos, con igualdad en el uso y el disfrute de las ciudades y los asentamientos humanos y reconoce que algunos actores han catalogado a esta visión como "el derecho a la ciudad".

Esta agenda, sumada al Acuerdo de París sobre cambio climático y a la Agenda 2030 con sus diecisiete Objetivos de Desarrollo Sostenible (ODS), constituyen compromisos internacionales que orientan las políticas de desarrollo de los Estados miembros de la Organización de las Naciones Unidas (ONU), y deberán ejecutarse durante los próximos años mediante amplias alianzas entre los distintos niveles de gobierno, el sector privado y la sociedad civil.

Considerando que la ciudadanía sería la principal beneficiaria de la concreción de estas agendas, su participación debería ser un elemento clave para

* Ponencia presentada en el I Congreso Interinstitucional de Ciencias Políticas y Relaciones Internacionales, organizado por CIPRI.

** Abogado, Pontificia Universidad Católica del Ecuador. Máster en Ciencias de la Población y el Desarrollo, Universidad Católica de Lovaina, Bélgica. Máster en Derechos Humanos y Democracia, Universidad Andina Simón Bolívar, Sede Ecuador. Docente adjunto, Escuelas de Derecho y Relaciones Internacionales, Universidad Internacional del Ecuador; jadavalosgo@internacional.edu.ec〉. 
lograr su adecuada implementación. Si bien se habla de la creación de la NAU y de los ODS como los procesos más participativos de la historia de las Naciones Unidas, con la realización de amplias consultas a todos los sectores, su proceso de territorialización necesitará de mecanismos de participación aún más contundentes y de un esfuerzo descentralizado que permita el accionar de más sujetos en la construcción colectiva en las fases de adaptación, implementación y seguimiento de las mismas.

Por esta razón, se considera que la participación ciudadana puede servir en una doble vía: como una herramienta de apropiación de la Agenda 2030 y la NAU dentro de las subjetividades de los ciudadanos y de sus prácticas cotidianas; $y$, como una herramienta de localización, al alimentar las agendas con la perspectiva territorial, en un diálogo constante entre necesidades locales y visiones globales.

\section{La Conferencia Hábitat III y la Nueva Agenda Urbana}

Hábitat es la conferencia internacional más importante sobre vivienda y desarrollo urbano sostenible. se realiza cada 20 años y es liderada por la agencia de Naciones Unidas UN Hábitat. En su tercera edición, esta conferencia fue organizada por el Ministerio de Desarrollo Urbano y Vivienda de Ecuador, en la ciudad de Quito, siendo la primera vez que una conferencia de esta envergadura se realizaba en América Latina. Este hito representa un reconocimiento para el aporte de esta zona del planeta a los procesos de transformación en la urbanización, pobreza y lucha contra la desigualdad. ${ }^{1}$

La Conferencia de las Naciones Unidas sobre los Asentamientos Humanos realizada en Vancouver (Canadá) en 1976, conocida como en la actualidad Hábitat I, concentró sus esfuerzos en torno la temática de los asentamientos informales, emitiendo la Declaración de Vancouver sobre la vivienda adecuada y asentamientos humanos. Veinte años después, en el año 1996, se llevó a cabo en Estambul (Turquía) la Cumbre de las Ciudades o Hábitat II, donde se adoptó la Declaración de Estambul y la Agenda Hábitat en la que tuvo protagonismo el debate alrededor del derecho a la vivienda digna.

1. Pamela Olmedo, La sociedad civil en el camino a Hábitat III, (Quito: ILDIS, 2016). 
Según el texto de la NAU, aprobada en Hábitat III en el tiempo transcurrido desde estas dos conferencias "se ha constatado mejoras en la calidad de vida de millones de habitantes de las zonas urbanas, entre ellos los habitantes de los barrios marginales y los asentamientos informales". ${ }^{2}$ No obstante, la misma introducción de la NAU señala que:

la persistencia de múltiples formas de pobreza, las desigualdades crecientes y la degradación ambiental siguen siendo uno de los principales obstáculos para el desarrollo sostenible en todo el mundo, siendo con frecuencia la exclusión social y económica y la segregación espacial una realidad irrefutable en las ciudades y los asentamientos humanos. ${ }^{3}$

De esta manera, según el texto de la NAU, sería "necesario aprovechar las oportunidades que presenta la urbanización como motor impulsor de un crecimiento económico sostenido e inclusivo, el desarrollo social y cultural y la protección del medio ambiente, así como de sus posibles contribuciones al logro de un desarrollo transformador y sostenible". ${ }^{4}$ Esto ya que

al reorientar la manera en que se planifican, se diseñan, se financian, se desarrollan, se administran y se gestionan las ciudades y los asentamientos humanos [...] ayudará a poner fin a la pobreza y al hambre en todas sus formas y dimensiones, reducir las desigualdades, promover un crecimiento económico sostenido, inclusivo y sostenible, lograr la igualdad de género y el empoderamiento de todas las mujeres y las niñas a fin de aprovechar plenamente su contribución vital al desarrollo sostenible, mejorar la salud y el bienestar humanos, fomentar la resiliencia y proteger el medio ambiente. ${ }^{5}$

\section{El rol de la sociedad civil en Hábitat III}

La sociedad civil puede ser entendida como la esfera organizada de la vida social. Está entonces conformada por actores colectivos o individuales que expresan y representan diversos intereses, imaginarios, prácticas, discursos y valores y que efectúan demandas al Estado para la consecución de fines y/o bienes públicos. ${ }^{6}$

2. ONU, Nueva Agenda Urbana (Quito: Secretariado Hábitat III, 2017), 1.

3. Ibíd.

4. Ibíd.

5. Ibíd.

6. Juan Carlos Portantiero. "Gramsci, lector de Maquiavelo". En Tomás Varnagy, Fortuna y virtud en la República Democrática. Ensayos sobre Maquiavelo (Buenos Aires: CLACSO, 2000): 149-154. 
Estos actores, necesariamente se insertan en un contexto determinado sobre el cual pretenden tener un grado de incidencia. El contexto al que se hace referencia, por lo general está encuadrado en el campo de lucha para la definición, el diseño y la ejecución de las políticas públicas, que pueden entenderse como la reacción de los Estados y gobiernos para solucionar los problemas y conflictos de la sociedad.

Ahora bien, cabe señalar que esta reacción debe tener un contenido técnico, que le permita entenderse como la mejor respuesta a un problema, siempre se enmarca en un contexto político de lucha de intereses, donde existirán ganadores y perdedores. La sociedad civil tiene entonces un rol trascendental en el proceso de formulación y ejecución de las políticas, que se puede reflejar en momentos de investigación, activismo, movilización, etc., donde se convierte en vocera de las aspiraciones y demandas ciudadanas, y con un profundo conocimiento sobre la realidad, es capaz de proponer mecanismos y soluciones a los problemas que se plantean diariamente.

En ese sentido, considerando la importancia de la participación en la definición e implementación de políticas públicas, este sería un elemento clave para implementar a nivel local la Nueva Agenda Urbana. Por esta razón, "[L] os actores de la sociedad civil están llamados a ser entes activos en la construcción de sus ciudades (...) deben pensar no solo en discursos sino también en acciones concretas que permitan trazar una agenda y que establezcan hitos que se conquisten paulatinamente".

\section{Participación social en la construcción de la Nueva Agenda Urbana}

Con el fin de garantizar un proceso ampliamente participativo para la construcción de la NAU, el Secretariado de UN Hábitat fijó una hoja de ruta que pretendió abarcar una diversidad de temas y con la mayor cantidad de actores posibles.

Así, la referida hoja de ruta para la participación previa a la conferencia Hábitat III incluyó la realización de tres Comités Preparatorios (con espacio para consultas abiertas); seis reuniones temáticas y cuatro reuniones regionales. Resultado de una de estas reuniones fue la Declaración de Toluca, que

7. Olmedo, "La sociedad civil en el camino a Hábitat III". 
manifestó que "es un elemento clave para la determinación de la NAU, tanto a nivel nacional como local, la creación de espacios de participación efectiva para que la ciudadanía sea parte del desarrollo de su ciudad, siendo estos espacios herramientas para garantizar una buena gobernanza, cohesión social y consolidación democrática". 8

Adicionalmente, se constituyó la campaña World Urban Campaign: una red global de urbanistas que incluyó a miembros de la sociedad civil, ciudades y grupos de negocios, alineados con la agenda propuesta por UN Habitat. Finalmente, estableció la Asamblea General de Socios (GAP, por sus siglas en inglés) constituida por quince miembros representantes de los major groups y de otros grupos de interés.

En el marco de Hábitat III se llevaron a cabo 22 sesiones temáticas, 10 diálogos y 15 mesas redondas. Más allá de la existencia de estos espacios, la Nueva Agenda Urbana se aprobaría finalmente a la usanza tradicional, en reuniones intergubernamentales de acceso restringido a Jefes de Estado y delegaciones diplomáticas.

Cabe señalar que los textos preliminares siempre consideraron a la sociedad civil como un actor importante. Así, dentro del Draft Outcome Document, aprobado en septiembre de 2016 mediante un consenso alcanzado en el marco de una reunión informal de carácter intergubernamental en Nueva York, se estableció que la sociedad civil es un actor que contribuye a la gestión de políticas urbanas que las naciones deben liderar y se reconoció espacios que le corresponden para aportar sobre la agenda específica de cada nación, para la construcción de alianzas entre actores de las ciudades y para el monitoreo y evaluación de la implementación. ${ }^{9}$ En este documento se hizo hincapié en promover el sentido de propiedad de las ciudades por parte de sus habitantes, de manera que exista un mayor compromiso cívico y participación activa, así como más apoyo a la realización de reportes de avance. Todo esto con base en la idea de "ciudades para todos" que constituyó finalmente el pilar básico en la visión de la NAU.

8. ONU, Declaración de Toluca para Hábitat III América Latina y el Caribe (Toluca: Secretariado Hábitat III, 2016).

9. ONU, Hábitat III New Urban Agenda Draft Outcome Document for adoption in Quito (New York: Secretariado Hábitat III, 2016). 
Por otro lado, cabe destacar que el hecho de ser la ciudad de Quito la sede de Hábitat III motivó el interés de la ciudadanía y permitió la realización de varios espacios de participación, tanto oficiales como alternativos, donde participaron varios colectivos, movimientos y en general ciudadanos y ciudadanas que buscaron conocer sobre las temáticas a tratarse en la conferencia y establecer sus puntos de vista al respecto.

Así, desde la organización nacional del evento, se dio paso a foros urbanos nacionales y foros territoriales. Desde el Secretariado de Habitat III se convocó a una serie de eventos como desayunos urbanos; la Urban Journalism Academy; el foro de stakeholders o interesados; y finalmente, Hábitat III Village, una especie de laboratorio urbano en vivo que se realizó de forma paralela a la conferencia en distintas ubicaciones de la ciudad de Quito por primera vez en la historia de las conferencias Hábitat.

Finalmente, desde el ámbito de la ciudadanía, se constituyeron varias entidades alrededor de las temáticas que se discutirían en Hábitat III, como la Plataforma de la Sociedad Civil por el Hábitat y el Derecho a la Ciudad, la Red Académica para Estudios de la Ciudad, el YoutHab Conference, el JapiAwer Urbano, entre otros. Incluso existieron espacios como Resistencia Hábitat 3 y Hacia un Hábitat 3 Alternativo, que no necesariamente estuvieron alineados con la agenda de Hábitat III pero aprovecharon la coyuntura para reunir a un grupo importante de personas para debatir temas urbanos.

Cabe resaltar que los temas tratados fueron variados. Por ejemplo, en el marco de la "Discusión temática de la NAU: camino a Hábitat III", los principales temas de interés en el foro fueron: inclusión y consideración de grupos vulnerables en las ciudades; normativa y planificación de la ciudad y elementos clave (suelo, infraestructura urbana y vivienda); tratamiento del sector económico informal; culturas urbanas; cambio climático urbano; formación de ciudades inteligentes; finanzas municipales, entre otros. ${ }^{10}$

10. Olmedo, "La sociedad civil en el camino a Hábitat III". 


\section{El derecho a la ciudad en el marco de la NAU}

Henri Lefebvre, en el año 1968, establecía su idea del derecho a la ciudad a raíz del impacto negativo debido a la conversión de la ciudad en mercancía al servicio de la acumulación de capital, llegándose a señalar que este derecho es "el usufructo equitativo de las ciudades dentro de los principios de sustentabilidad y justicia social"."

Con el paso de los años, autores como David Harvey han retomado este concepto y han señalado que el derecho a la ciudad:

Es el derecho de toda persona a crear ciudades que respondan a las necesidades humanas, ya que todo el mundo debería tener los mismos derechos para construir los diferentes tipos de ciudades que queremos. El derecho a la ciudad entonces no es simplemente el derecho a lo que ya está en la ciudad, sino el derecho a transformar la ciudad en algo radicalmente distinto. ${ }^{12}$

En definitiva, el derecho a la ciudad retoma la expresión fundamental de los intereses colectivos, sociales y económicos en la ciudad, en especial de los grupos vulnerables y desfavorecidos, respetando las diferentes culturas urbanas y el equilibrio entre lo urbano y lo rural. Algunos de los principios que conforman el contenido de este derecho son: el ejercicio pleno de la ciudadanía, la gestión democrática de la ciudad, la función social de la ciudad y de la propiedad y la protección especial a grupos y personas en situación vulnerable.

En la Constitución ecuatoriana del 2008 el derecho a la ciudad se establece en el artículo 31, que señala:

Las personas tienen derecho al disfrute pleno de la ciudad y de sus espacios públicos, bajo los principios de sustentabilidad, justicia social, respeto a las diferentes culturas urbanas y equilibrio entre lo urbano y lo rural. El ejercicio del derecho a la ciudad se basa en la gestión democrática de esta, en la función social y ambiental de la propiedad y de la ciudad, y en el ejercicio pleno de la ciudadanía. ${ }^{13}$

Por otro lado, a nivel internacional, la Carta Mundial del Derecho a la Ciudad expedida en el año 2004 reconoce que el derecho a la ciudad es un "de-

11. Henri Lefebvre, Le Droit à la ville (Paris: Ed. du Seuil, 1968).

12. David Harvey, Rebel Cities: From the Right to the City to the Urban Revolution (London: Verso, 2012).

13. Constitución de la República del Ecuador [2008] cap. segundo "Derechos del buen vivir", sec. sexta "Hábitat y vivienda", art. 31 ([Quito]: Asamblea Nacional, 2011), 33. 
recho colectivo de los habitantes de las ciudades, en especial de los grupos vulnerables y desfavorecidos, que les confiere legitimidad de acción y de organización, basado en sus usos y costumbres, con el objetivo de alcanzar el pleno ejercicio del derecho a la libre autodeterminación y un nivel de vida adecuado." 14

El texto de la Nueva Agenda Urbana recoge el derecho a la ciudad en el párrafo 11, en lo que se titula como "nuestro ideal común", en los siguientes términos:

11. Compartimos el ideal de una ciudad para todos, refiriéndonos a la igualdad en el uso y el disfrute de las ciudades y los asentamientos humanos y buscando promover la inclusividad y garantizar que todos los habitantes, tanto de las generaciones presentes como futuras, sin discriminación de ningún tipo, puedan crear ciudades y asentamientos humanos justos, seguros, sanos, accesibles, asequibles, resilientes y sostenibles y habitar en ellos, a fin de promover la prosperidad y la calidad de vida para todos. Hacemos notar los esfuerzos de algunos gobiernos nacionales y locales para consagrar este ideal, conocido como "el derecho a la ciudad", en sus leyes, declaraciones políticas y cartas. ${ }^{15}$

De esta manera, el texto de la NAU recoge la idea del derecho a la ciudad, aunque no con la fuerza que muchas delegaciones y organizaciones de la sociedad civil hubieran deseado.

\section{Los retos de la sociedad civil}

La reconfiguración de las relaciones entre los actores clásicos de la cooperación internacional hace que ya no sean solamente los Estados los protagonistas de la implementación de las agendas internacionales. El sector privado, los gobiernos locales y los movimientos y organizaciones sociales se consideran actores con enorme trascendencia, lo que representa una gran oportunidad para que la sociedad civil protagonice la construcción de las ciudades entendidas como campus en disputa.

En este sentido, durante la Segunda Asamblea Mundial de Gobiernos Locales y Regionales, celebrada en Quito el día previo a la inauguración de la Conferencia Hábitat III, el ex secretario general de la ONU, Ban Ki Monn, re-

14. ONU Hábitat, "Carta Mundial del Derecho a la Ciudad, 2004”. Revista Paz y Conflictos, No. 5 (2012): 184-196.

15. ONU, Nueva Agenda Urbana (Quito: Secretariado Hábitat III, 2017). 
conoció que el sistema de gobernanza global ya no era el más adecuado para abordar los desafíos globales, ya que la función catalizadora de los gobiernos locales entre todos los actores es fundamental para responder a las necesidades de las comunidades. ${ }^{16}$

La participación de la sociedad civil ha demostrado ser un factor clave para el éxito de la aplicación local de políticas de desarrollo globales. En efecto, autores como David Harvey ${ }^{17}$ afirman que toda política pública debe tener raíces en las circunstancias locales y que se deben comenzar a configurar vínculos firmes y activos entre las organizaciones locales y los distintos niveles de gobierno, en términos de su acción política.

Así, en la actualidad los grandes desafíos globales, como el cambio climático y el calentamiento global, la pobreza y la desigualdad o la grave crisis energética, son problemas que se viven en la cotidianeidad de la ciudad y gran parte de su origen está en las relaciones del entorno urbano. En Ecuador, el porcentaje de personas que viven en ciudades ha aumentado del 34\% en los años 60 al 64\% en el año 2015. ${ }^{18}$ A nivel global el 75\% del consumo de energía y el $80 \%$ de las emisiones de gases que causan el calentamiento global provienen de las ciudades.

Las ciudades ecuatorianas producen toneladas y toneladas de desechos, todavía utilizan sistemas de transporte basados en combustibles fósiles en su gran mayoría y siguen generando a diario problemas de segregación social. Sin embargo, es también en las ciudades donde surgen a diario increíbles iniciativas que utilizan el poder, la innovación social y la creación colectiva para construir ciudades inclusivas, resilientes y sostenibles.

En la ciudad de Quito, por ejemplo, han surgido proyectos de reciclaje inclusivo para reducir la cantidad de desechos que terminan en los rellenos sanitarios, es aquí también donde existen colectivos ciudadanos luchando para mejorar nuestra política de transporte y promoviendo el uso de la bicicleta como alternativa de movilidad sostenible, y donde trabajan a diario organizaciones que buscan generar mejores condiciones para los grupos más vulnerables.

16. Fernando Casado Cañeque, "La revancha de los alcaldes", El País (Madrid), 10 de diciembre de 2016, edición digital.

17. Harvey, "Rebel Cities: From the Right to the City to the Urban Revolution".

18. Banco Mundial, "Naciones Unidas perspectivas de la urbanización mundial 1960-2017”, 〈https://datos. bancomundial.org/indicador/SP.URB.TOTL.IN.ZS〉. 
Si bien existen retos como la institucionalización de la participación ciudadana, el desconocimiento de los espacios generados y el creciente uso de las redes sociales, que amplían la acción colectiva y rompen fronteras y horizontes pero desmotivan el activismo off line, las ciudades representan también claras oportunidades. Los emprendimientos innovadores, la participación ciudadana o la toma de decisiones sencillas e individuales como separar la basura, contribuyen para hacer de la ciudad un espacio donde sus habitantes puedan construir proyectos de vida que sean social, ambiental y económicamente sostenibles.

La ciudad es un espacio colectivo. Es la suma de individualidades y subjetividades y todo lo que sucede en ella, afecta a todos sus habitantes de diferentes maneras. Por ello, fortalecer el tejido social, crear sentido de comunidad y crear espacios de participación son caminos hacia la construcción de las ciudades y comunidades sostenibles y resilientes que busca la Nueva Agenda Urbana.

\section{Bibliografía}

Banco Mundial. "Naciones Unidas perspectivas de la urbanización mundial 1960-2017", 〈https://datos.bancomundial.org/indicador/SP.URB.TOTL.IN.ZS〉.

Casado Cañeque, Fernando. "La revancha de los alcaldes", El País (Madrid), 10 de diciembre de 2016, edición digital.

Constitución de la República del Ecuador [2008] cap. segundo "Derechos del buen vivir", sec. sexta "Hábitat y vivienda", art. 331 [Quito]: Asamblea Nacional, 2011.

Harvey, David. Rebel Cities: From the Right to the City to the Urban Revolution. London: Verso, 2012.

Lefebvre, Henri. Le Droit à la ville. Paris: Ed. du Seuil, 1968.

Olmedo, Pamela. La sociedad civil en el camino a Hábitat III. Quito: ILDIS, 2016.

ONU. Declaración de Toluca para Hábitat III América Latina y el Caribe. Toluca: Secretariado Hábitat III, 2016.

- Hábitat III New Urban Agenda Draft Outcome Document for adoption in Quito. New York: Secretariado Hábitat III, 2016.

—. Nueva Agenda Urbana. Quito: Secretariado Hábitat III, 2017.

ONU Hábitat. "Carta Mundial del Derecho a la Ciudad, 2004". Revista Paz y Conflictos, No. 5 (2012): 184-196.

Portantiero, Juan Carlos. "Gramsci, lector de Maquiavelo". En Tomás Varnagy. Fortuna y virtud en la República Democrática. Ensayos sobre Maquiavelo, 149-154. Buenos Aires: CLACSO, 2000. 